\title{
A Neural Genetic Hybrid Model for Eigenstructure Allocation in the LQR Project in DFIG
}

\author{
Ivanildo Abreu \\ State University of \\ Maranhão UEMA \\ University City Paulo VI \\ $\mathrm{s} / \mathrm{n}$ - St. Louis
}

\author{
Rildenir Silva \\ State University of \\ Maranhão UEMA \\ University City Paulo VI \\ $\mathrm{s} / \mathrm{n}$ - St. Louis
}

\author{
Luan Pereira \\ State University of \\ Maranhão UEMA \\ University City Paulo VI \\ $\mathrm{s} / \mathrm{n}$ - St. Louis
}

\begin{abstract}
A hybrid neuronal genetic model is proposed with the objective of solving the Riccati Algebraic Equation (RAE) that is associated to the restricted optimization structure of the Linear Quadratic Regulator (LQR) problem. The application of this hybrid model of artificial intelligence will be performed in a wind power generation system, in particular, the double fed induction generator (DFIG). For this, a recurrent neural network with multiple layers is used where its performance is realized by metrics of the norm of infinity associated with RAE and energy surfaces as a function of the positive definite symmetric matrix and the Cholesky factor.
\end{abstract}

\section{General Terms}

RAE, LQR, hybrid model, wind power, DFIG

\section{Keywords}

Artificial Neural Networks (RNA), genetic algorithm (GA), DFIG, LQR.

\section{INTRODUCTION}

The Double Fed Induction Generator (DFIG) is a relatively new technology and provides a strong insertion in the market due to its application in wind power generation. In Brazil, the first experience with the generation and use of wind energy was through a wind turbine installed in the Fernando de Noronha archipelago in the 90's. Over the years, several wind farms have been installed throughout Brazil in which are installed wind turbines that are distinguished in two types:

- Generator driven directly by the Turbine shaft;

- Generator driven by the Turbine shaft, using multiplier.

Wind Energy can be converted into electrical energy through aero generators which are systems that harness wind energy through the propellers, converting it into mechanical energy which in turn is converted into electrical energy through an electricity generator.

The principle of operation of the DFIG is identical to AC electric generators, but has more relevant features that allow them to run at speeds slightly above or below their natural synchronous speed. These generators are widely used in large wind turbines of varying speed, due to the speed of the wind being variant with the time. Figure (1) illustrates a DFIG installation model:

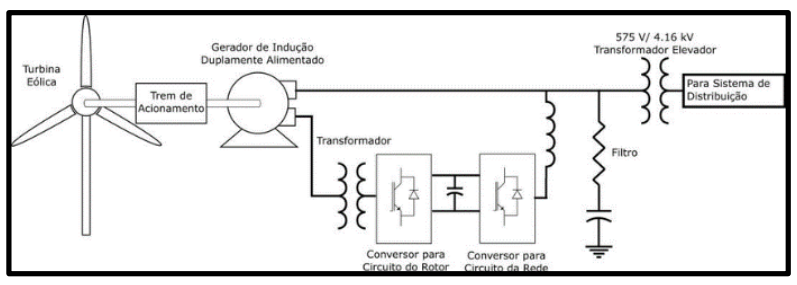

Figure 1: DFIG model coupled to the turbines

Induction generators, in particular those with winding rotor applied in wind power systems, can be found in two types of basic configurations:

- The torque or slip is controlled by power electronics in the rotor circuit, characterized by the variation of the rotor resistance;

- The other configuration consists of the connection of a circuit that draws power through the rotor (DFIG).

According to (Hansen, 2003), the operating principle of the DFIG is based on the slip that can be determined by $s=$ $\frac{\omega_{s}-\omega_{m}}{\omega_{s}}$ which represents the power flow.

The demand for new renewable energy sources for the development of increasingly efficient energy generation systems has been made possible by new computational techniques to optimize these activities. In this sense, we will approach in this work computational techniques based on artificial intelligence such as: Artificial neural networks and genetic algorithms in order to synthesize optimal control systems related to the Linear Quadratic Regulator (LQR) problem.

In this context, Myoen-Song Choi (1996) lists the main areas of application where control systems models are concentrated, where we will find the LQR project fundamentals such as industrial sector, power generation, noise attenuation, Machines of direct current, among others. According to (Pinto, 2007), the wide use of the LQR project has brought great results in wind power systems that use as power generation the dual-fed induction generators.

This article is organized in sections according to the following sequence: Section 2 presents the methodology of this study. In Section 3, presents the intelligent control $L Q R$ linked to the structure of the genetic algorithm that will function to obtain the weights matrices $Q$ and $R$. In Section 4, presents the structure of the recurrent neuronal network that will solve the RAE providing a matrix as Solution, and this matrix is symmetric and definite positive. In Section 5, the turbine generator scheme connected to the LQR controller and its linearized models is shown. Section 6 presents the computational results applied to the turbine generator model. 
And in Section 7, the conclusions, comments and expectations of the intelligent $L Q R$ project are presented.

\section{METHODOLOGY}

These goals will be achieved through the use of Artificial Intelligence paradigms, in particular, genetic algorithms and neural networks. The first one is responsible for the allocation of eigenvalues and eigenvectors, while the second justifies the solution of the Riccati Algebraic Equation. The convergence of GA and RNA stability are guaranteed.

\section{INTELLIGENT CONTROLLER}

Here presents a hybrid neuronal genetic model to be applied to solve the LQR problem. According to (Wang; Wu, 1998) these evolutionary models come from studies using a genetic algorithm to select the $Q$ and $R$ weighting matrices and a recurrent artificial neural network that solves the Riccati Algebraic Equation. Figure (2) illustrates a scheme for these procedures:

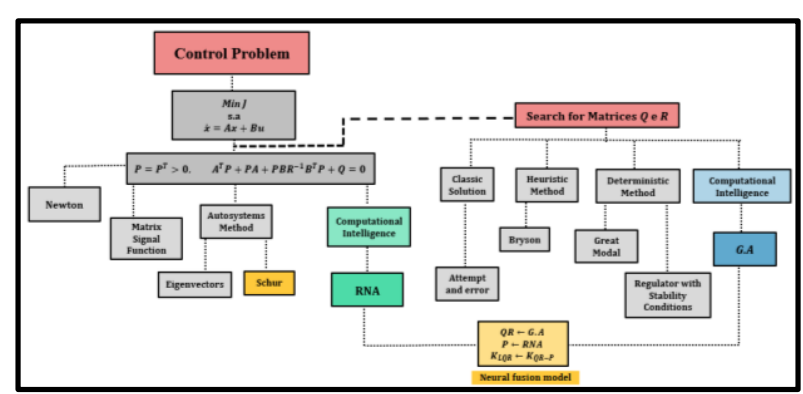

Figure 2: Intelligent control scheme

\subsection{Classical approach LQR}

This approach covers the formulation and solution of the Linear Quadratic Regulator problem, as well as the Schur method, which solves RAE. In this classic analysis of LQR, the input vector $u$ is sought which aims to minimize a cost function $J$ with some restrictions for the dynamic model of the system.

According to some authors, one of the advantages of the linear quadratic regulator is the guaranteed stability margin where the phase margin is $60^{\circ}$ and the gain margin is infinite, thus having a stability quality.

The Linear Quadratic Regulator is modeled by a quadratic performance index and has some constraints on its linear and time invariant state cost function as follows:

$$
J\left(t_{0}\right)=\frac{1}{2} \int_{t_{0}}^{T}\left[x^{T} Q x+u^{T} R u\right] d t
$$

s.a

$$
\dot{x}=A x+B u
$$

at where $x \in R^{n}, u \in R^{m}$, the pair of matrices $\boldsymbol{A}$ and $\boldsymbol{B}$ is controllable, the pair A and C is observable, $Q \geq 0, R>0$ and all are symmetrical. Respectively, The matrices $\boldsymbol{Q} \in R^{n x n}$ and $\boldsymbol{R} \in R^{n x m}$ are definite and semi-definite positive matrices.

According to (Athans, 1966), some variational calculation methods can be used to solve the optimization problem that is inherent to the optimization structure of equations (1) and (2). Such methods will provide the control law that will minimize the cost function $J$ and, at the same time, obey the constraints of the vector $\dot{x}$ related to the optimization structure that follows,

$$
u=K x
$$

At where $K=-R^{-1} B^{T} P$ is the gain of the controller. The gain $K$ depends on the matrix $P$ which is the solution of EAR given by:

$$
A^{T}+P A-P B R^{-1} B^{T} P+Q=0
$$

with $P$ symmetric and positive definite.

\subsubsection{RAE and the $L Q R$}

According to (Laub, 1979), the Riccati Algebraic Equation (RAE) can be solved by reducing the Hamiltonian matrix. This matrix has the following structure:

$$
=\left[\begin{array}{cc}
A & -B R^{-1} B^{T} \\
-Q & -A^{T}
\end{array}\right]
$$

Using the Schur method to obtain a quasi-triangular shape using orthogonal similarity transformations, we can find an orthogonal matrix $\mathrm{U}$, which transforms the Hamiltonian matrix $H$ into the real Schur form, that is:

$$
T=W^{T} H W=\left[\begin{array}{cc}
T_{11} & T_{12} \\
0 & T_{22}
\end{array}\right]
$$

Where the blocks $T_{11}$ e $T_{22}$ are quasi-triangular upper matrices. $W$ is a matrix partitioned into four dimension blocks $n \times n$. If the matrix $W_{11}$ are non-singular matrices, the solution of the Riccati Algebraic Equation is definite positive, that is:

$$
P=W_{21} W_{11}^{-1}
$$

The matrix $T_{11}$ has eigenvalues that represent the closed-loop spectrum of the matrix $\left(\boldsymbol{A}-\boldsymbol{B} \boldsymbol{R}^{-\mathbf{1}} \boldsymbol{B}^{\boldsymbol{T}} \boldsymbol{P}\right)$.

Another important consideration is that the polar region of the optimal closed-loop system is due to the following relationship

$$
\left(A-B R^{-1} B^{T} P\right)=W_{11} T_{11} W_{11}^{-1}
$$

\subsection{Genetic Algorithm Architecture}

For the proposed GA model, it has been as weights matrices $\boldsymbol{Q}$ e $\boldsymbol{R}$ which establishes the free parameters of the LQR project, since they will be used to adjust the optimal gain of the controller. The main objective is the search for the weighting matrices that form the quadratic performance index $J$. Given this context, a nonlinear programming problem will arise to optimize the $Q$ and $R$ weighting matrices in terms of computational evolution algorithms and data structures, according to the following equation:

$$
\min _{Q, R} \sum_{i=1}^{n} p_{i} s_{i}(Q, R)
$$

s. $a$

$$
\begin{array}{ll}
s_{i}(Q, R) \leq 1, & i=1, \ldots, n \\
\lambda_{e i} \leq \lambda_{c i}(Q, R) \leq \lambda_{d i}, & i=1, \ldots, n
\end{array}
$$

At where $p_{i}$ are the weights related to the sensitivity $s_{i} / \epsilon_{i}$ normalized with respect to ith project specification where we should have $\epsilon_{i}>0$. In this model, the genetic representation of the $Q$ and $R$ matrices is approached through the operations between the chromosomes arriving at the performance evaluations. According to (Silva, 2008), the chromosomal model that represents the weights matrices $Q$ and $R$ is presented below: 


$$
Q R_{z}=\bigcup_{i, j=1}^{n} q_{i, j} \wedge \bigcup_{j, i=1}^{m} r_{i, j}
$$

com $\mathrm{i} \leq \mathrm{j}, \quad \mathrm{z}=1, \ldots, \mathrm{n}_{\text {ind }}, q_{i, j}$ and $r_{i, j}$ represent the genes of the matrices $Q R_{z}$ and the term $n_{\text {ind }}$, characterizes the number of individual chromosomes of a population. The random generation model of the search matrices is given by:

$$
q_{i, j}= \begin{cases}P_{Q, \alpha}+P_{Q, \beta} k_{Q_{i, j},} & i=j \\ P_{Q, \lambda} k_{Q_{i, j}} & i \neq j \\ i, j=1, \ldots, n & \end{cases}
$$

With $P_{Q, \alpha}$ and $P_{Q, \beta}$, respectively, are fixed and variable parameters belonging to the elements of the diagonal $q_{i, j}$. The model of generation of the individual for the matrix $R$ is identical to the model of generation of the individual for the matrix $Q$, as it shows the equation that follows:

$$
r_{i, j}= \begin{cases}P_{R, \alpha}+P_{R, \beta} k_{R_{i, j}}, & i=j \\ P_{R, \lambda} k_{R_{i, j}} & i \neq j \\ i, j=1, \ldots, n & \end{cases}
$$

The selection operation is based on the values of the performance function.

\section{ARCHITECTURE OF RNAR}

For the synthesis of the LQR project, the new formulation can be considered as an unrestricted optimization problem, so that, given the matrices $A, B, Q$ and $R$, we intend to find the matrices $P$ and $L$ in order to minimize an energy function and, given by the new formulation:

$$
\min _{P, L} \xi\left(P S P-A^{T} P-P A-Q+L L^{T}-P\right)
$$

where $S=B R^{-1} B^{T}$. Considering that the activation function $\xi_{i j}=\mathbb{R} \rightarrow \mathbb{R}$, is a convex function bounded at the bottom, then it is shown that the sum of the Riccati Algebraic Equation, using Cholesky's factorization, can be rewritten as follows:

$$
\begin{gathered}
\sum_{k=1}^{n} \sum_{l=1}^{n} p_{i k} s_{k l} p_{l j}-\sum_{k=1}^{n}\left(a_{k i} p_{k j}+p_{i k} a_{k j}\right)-q_{i j} \\
+\sum_{k=1}^{\operatorname{migi,j\} }} l_{i k} l_{j k}-p_{i j}
\end{gathered}
$$

The dynamic equations that symbolize the recurrent neural network, being, $i=1, \ldots, n$ and $j=1, \ldots, n$ are given by:

$$
\begin{gathered}
\frac{d v_{i j}}{d t}=-\eta_{v}\left\{\sum_{k=1}^{n} \sum_{l=1}^{n}\left[v_{i k} s_{k l} u_{l j}+u_{i k} s_{k l} v_{l j}\right]\right. \\
\left.-\sum_{k=1}^{n}\left[a_{i k} u_{k j}+u_{i k} a_{j k}\right]-y_{i j}\right\} \\
\frac{d z_{i j}}{d t}=-\eta_{z} \sum_{k=j}^{n} y_{i k} z_{k j}, \quad i \geq j \\
u_{i j}=f_{i j}\left\{\sum_{k=1}^{n} \sum_{l=1}^{n} v_{i k} s_{k l} v_{l j}-\sum_{k=1}^{n}\left(a_{k i} v_{k j}+v_{i k} a_{k j}\right)\right. \\
\left.+q_{i j}\right\}
\end{gathered}
$$

$$
y_{i j}=f_{i j}\left[\sum_{k=1}^{\min \left[\operatorname{Iin}_{i j}\right\}} z_{i k} z_{j k}-v_{i j}\right]
$$

Matricially, these equations are expressed as:

$$
\begin{gathered}
\frac{d V}{d t}=-\eta_{\nu}\left[V(t) S U(t)+U(t) S V(t)-A U(t)-U(t) A^{T}\right. \\
-Y(t)] \\
\frac{d Z(t)}{d t}=-\eta_{Z} Y(t) Z(t) \\
U(t)=F\left[V(t) S V(t)-A^{T} V(t)-V(t) A+Q\right] \\
Y=F\left[Z(t) Z(t)^{T}-V(t)\right]
\end{gathered}
$$

Where the matrices $U=\left[u_{i j}\right] \in \mathbb{R}^{n x n}, V=\left[v_{i j}\right] \in \mathbb{R}^{n x n}$ e $Y=\left[y_{i j}\right] \in \mathbb{R}^{n x n}$, are square matrices activation states, the matrix $Z=\left[z_{i j}\right] \in \mathbb{R}^{n x n}$ is of the lower triangular type of the activation states. We have as parameters of the project the conditions $V(0)=V(0)^{T}, Y(0) \neq 0, \eta_{v}>0$ and $\eta_{z}>0$. The matrix $F=f_{i j}$ is symmetric and belongs to the activation states, however, being $F$ symmetrical, then $V(t), U(t)$ and $Y(t)$ will also be. The fact that these matrices are symmetrical enables the reduction of the number of neurons used in the neural network construction.

In the proposed neural network architecture, there are four bidirectionally connected layers, where $V(t)$ is the output layer, $U(t)$ is the input layer, and $Y(t)$ and $Z(t)$ are two hidden layers that perform the interconnection between $P$ and $U$. The matrices $V(t), U(t)$ and $Y(t)$ are square characterizing layers of order $n x n$. The matrix $V(t)$ represents the computational result of $P$ and the state matrix $Z(t)$ represents $L$ which is the Cholesky factor of $P$. The matrix $P$, presented in equation (12) represents the solution of the RAE.

\subsection{Stability Analysis}

The stability of the recurrent neural network proposed here and analyzed in more detail in (Wang; Wu, 1998) is necessary since it is a continuous nonlinear dynamic system in time. This stability can be analyzed by using the direct Lyapunov method given by the following theorem:

Theorem 1. If every activation function is continuous, at least part-differentiable, and still non-decreasing, that is:

$$
\begin{gathered}
\frac{d f_{i, j}(\xi)}{d \xi} \geq 0 \quad \text { with }-\infty<\xi<+\infty \text { and, } \\
f_{i, j}(\xi)=0 \text { if } \xi=0, \text { for } i, j=1,2 \ldots, n
\end{gathered}
$$

Then the RNAR is asymptotically stable assuming values in time tending towards infinity, symbolically, it has been:

$\forall \boldsymbol{V}(0)$ and $\boldsymbol{Z}(0) \exists \widetilde{\boldsymbol{Z}}$ and $\widetilde{\boldsymbol{V}}$ tal que $\lim _{t \rightarrow \infty} Z(t)=\widetilde{\boldsymbol{Z}}$ and $\lim _{t \rightarrow \infty} V(t)=\widetilde{\boldsymbol{V}}$.

This stability analysis of the neural network shows that the activation state transition generates a gradient flow which, in turn, minimizes the energy function $\boldsymbol{E}$.

\subsection{Solvability analysis}

Theorem 2. This theorem ensures a necessary and sufficient condition for the flow of the gradient function to converge to a symmetric and definite positive solution of Riccati Algebraic Equation, $P$, of any initial state (Wang; Wu, 1998). Theorem 2 tells us that, since every activation function is 
continuous, at least part-differentiable, and non-decreasing monotonic, that is:

$$
\begin{gathered}
\frac{d f_{i, j}(\xi)}{d \xi} \geq 0 \quad \text { with }-\infty<\xi<+\infty \text { and, } \\
f_{i, j}(\xi)=0, \text { if } \xi=0, \text { for } i, j=1, \ldots, n
\end{gathered}
$$

The RNAR solution, $V$, is always symmetric and positive definite for the Riccati equation of any symmetric initial state $V(0)$ and any non-zero initial state $Z(0)$, this is, $\forall V(0)=$ $V(0)^{T}, \forall Z(0) \neq 0, \tilde{V}=V$, If and only if, $\operatorname{rank}(\tilde{V} S-A)=$ $n$ and all diagonal elements in $\widetilde{\boldsymbol{Z}}$ are non-zero, this is, for all $i$, $\tilde{\mathbf{z}}_{i i} \neq 0$ for $i=1, \ldots, n$.

\section{TURBINE GENERATOR MODEL}

The wind turbine converts kinetic energy from the wind into rotational mechanical energy. According to (Vivas, 2016), the ideal turbine power, considering that there is possibility of converting all the kinetic energy of the wind is given by:

$$
P_{w i}=\frac{1}{2} \rho \Lambda v^{3}
$$

Being, $\boldsymbol{P}_{\boldsymbol{w} \boldsymbol{i}}$ the theoretical power; $\boldsymbol{\rho}$ the air density; $\boldsymbol{\Lambda}$ the area covering the turbine; And $v$ the wind speed.

A diagram of the turbine connections, with generator with coiled rotor and the controller connected to the converters, is shown in figure (3). One of the converters is connected to the network and the other to the rotor windings, so that they are interconnected through a circuit with capacitors and with a PWM pulse width modulation control. In the case of a generator set and power converters, there are several concepts for the interconnection of the double-fed induction generators to the wind turbines.

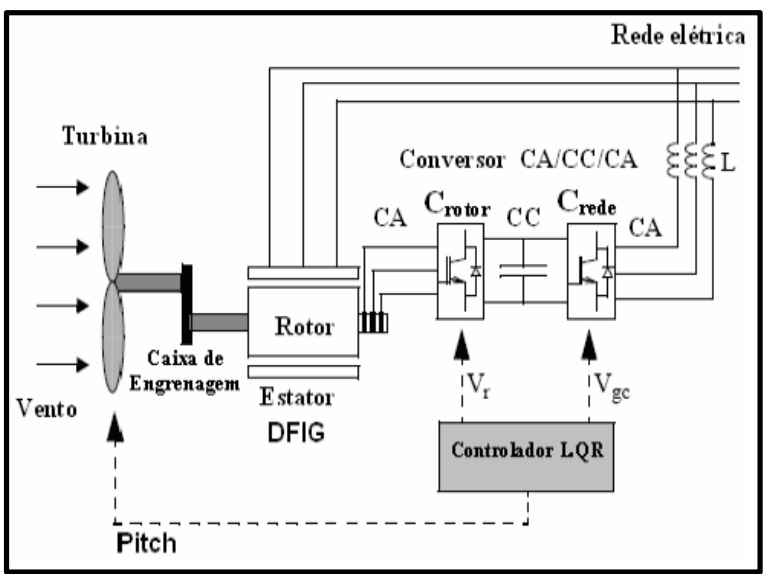

Figure 3: DFIG model connected to $L Q R$ controller.

As shown in figure (3), in this section, presents the linearized turbine generator model, in which linear systems theory can be used to analyze dynamics, stability, observability, frequency response and Robustness of the non-linear system, applying the intelligent LQR model to the DFIG system. As already mentioned in the previous sections, this method has the objective of searching GA matrices and determining the optimal gain of said controller, via RNAR. The following is a schematic of the linearized model:

$$
\begin{aligned}
& \Delta \dot{x}=A \Delta x+B \Delta u \\
& \Delta y=C \Delta x
\end{aligned}
$$

At where $\Delta x$ and $\Delta u$ are vectors of state and input, respectively, being:

$$
\begin{aligned}
& \Delta x=\left[\begin{array}{llll}
\Delta i_{d r} & \Delta i_{q r} \Delta \omega_{r} & \Delta i_{d} \Delta i_{q} \Delta V_{d c}
\end{array}\right]^{T} ; \\
& \Delta u=\left[\begin{array}{llll}
\Delta v_{d r} & \Delta v_{q r} \Delta T_{e} & \Delta v_{d} \Delta v_{q}
\end{array}\right]^{T}
\end{aligned}
$$

The state matrix $A$ is formed by block matrices such as: $A_{r}^{3 \times 3}$ e $A_{s}^{3 \times 3}$ which represent, respectively, the rotor and stator part. The matrix of control $B$, is constituted of matrices of block $B_{r}^{3 \times 2}$ e $B_{S}^{3 \times 2}$. According to equation (16), the state matrices take the forms:

$$
\begin{gathered}
A_{r}=\left[\begin{array}{ccc}
-\frac{R_{r}}{L_{r r} \sigma} & S_{0} \omega_{0} & 0 \\
-S_{0} \omega_{0} & -\frac{R_{r}}{L_{r r} \sigma} & 0 \\
0 & 0 & \mu
\end{array}\right] \\
B_{r}=\left[\begin{array}{ccc}
-\frac{1}{L_{r r} \sigma} & 0 & 0 \\
0 & -\frac{1}{L_{r r} \sigma} & 0 \\
0 & 0 & -\frac{1}{2 H}
\end{array}\right]
\end{gathered}
$$

$$
A_{S}=\left[\begin{array}{ccc}
-\frac{R}{L} & \omega_{0} & 0 \\
-W_{0} & -\frac{R}{L} & 0 \\
-\frac{v_{d 0}}{C V_{c c 0}} & -\frac{v_{q 0}}{C V_{c c 0}} & \frac{v_{d 0} i_{d 0}+v_{q 0} i_{q 0}}{C V^{2} c 0}
\end{array}\right]
$$

$$
B_{s}=\left[\begin{array}{cc}
\frac{1}{L} & \omega_{0} \\
0 & \frac{1}{L} \\
-\frac{i_{d 0}}{C V_{c c 0}} & -\frac{i_{q 0}}{C V_{c c 0}}
\end{array}\right]
$$

Where the coefficient $\mu$ belonging to the matrix $A_{r}$ is represented by the expression:

$$
\mu=-\frac{\frac{1}{2} \rho A v^{3} C_{p}(\lambda, \beta)}{2 H \omega_{r}^{2}}+\frac{\frac{1}{2} \rho A v^{3}}{2 H \omega_{r}} \cdot \frac{d C_{p}(\lambda, \beta)}{d \lambda} \cdot \frac{R_{p}}{v}
$$

According (Murata; Muyeen; Tamura, 2009), the term $C_{p}(\lambda, \beta)$ represents the power coefficient of fixed speed turbines that depends on the pitch angle $\beta$ of the shovel where $\lambda$ is the specific speed.

\section{COMPUTATIONAL RESULTS}

Based on the work of (Pinto, 2007), they follow the complete model and parameters of the wind turbine for application to DFIG and to the inverter that will be substituted in equations (23) and (24). After this application, the matrices of the state equation for the aforementioned system are obtained, investigated in relation to an operating point. The matrices are:

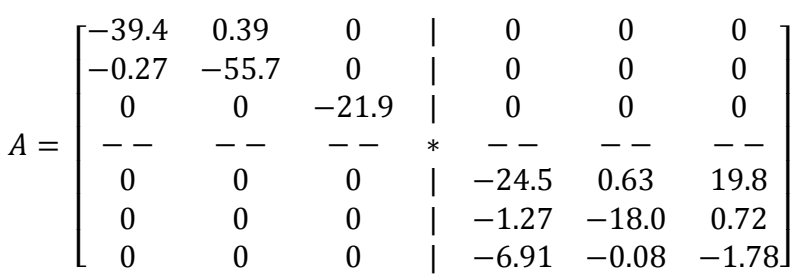

The control matrix is given by: 


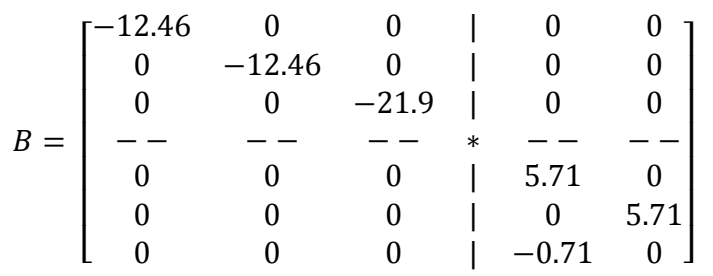

The construction of the output matrix $C$ can be seen in details in (Pinto, 2007), where we find:

$$
C=\left[\begin{array}{cccccc}
3.14 & 0 & 0 & 0 & 0 & 0 \\
0 & 4.51 & 0 & 0 & 0 & 0 \\
0 & 0 & -10.02 & 0 & 0 & 0 \\
0 & 0 & 0 & -42.66 & 0 & 0 \\
0 & 0 & 0 & 0 & 3.29 & 0 \\
0 & 0 & 0 & 0 & 0 & -3.06
\end{array}\right]
$$

As the proposed 6th order plant system is a MIMO system, the analysis using the Bode diagram becomes impracticable. We propose the use of the singular value decomposition (SVD), so that we can evaluate the behavior of the system in the frequency domain. Figure (4) shows the frequency response of the singular values.

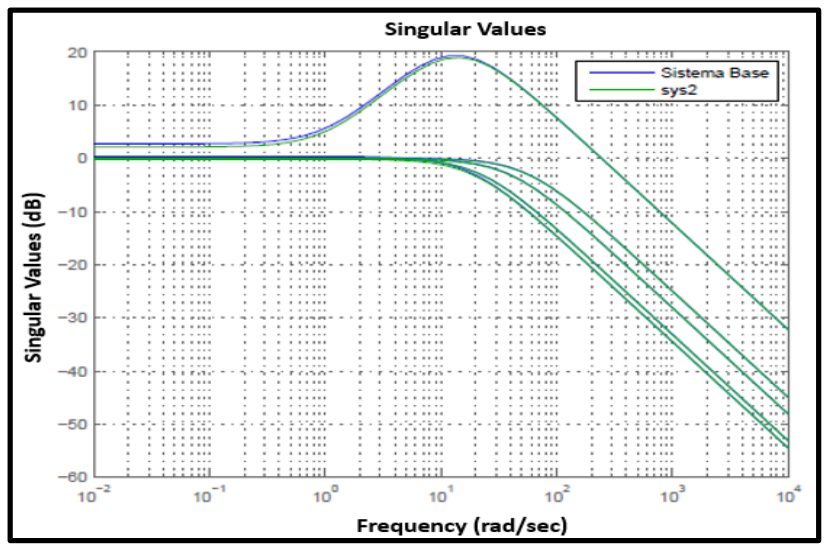

Figure 4: Singular Values of the 6th Order Wind Plant

It is observed in this figure the largest and smallest singular values in $d B$ as a function of the $\mathrm{rad} / \mathrm{sec}$ frequency of the base system and without controller and with the LQR.

Before performing the tests, it is determined the eigenvalues that verify that the system is stable,

$$
\text { [-39.42; -55.74; -13.37 } \pm j 2.60 ;-17.68 ;-21.95]
$$

The impulse response analysis, as shown in Figure (5), is performed with the purpose of evaluating more accurately the behavior of the DFIG plant dynamic system,

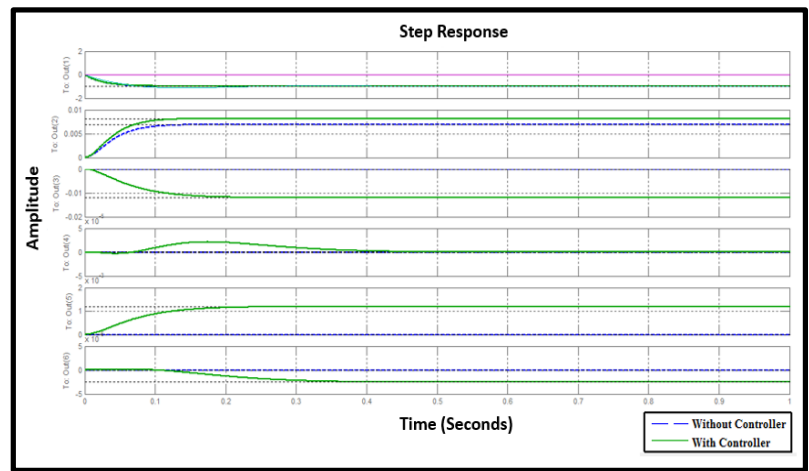

Figure 5: Response to the Impulse of the 6th Order System

\subsection{Selection of $Q$ and $R$ matrices}

This research uses a genetic algorithm to select the matrices $Q$ and $R$, where $Q$ represents the state and $R$ is the control matrix. Figure 6 shows the result of normalized final sensitivity for the selection of a group of twenty generations for each population of individuals:

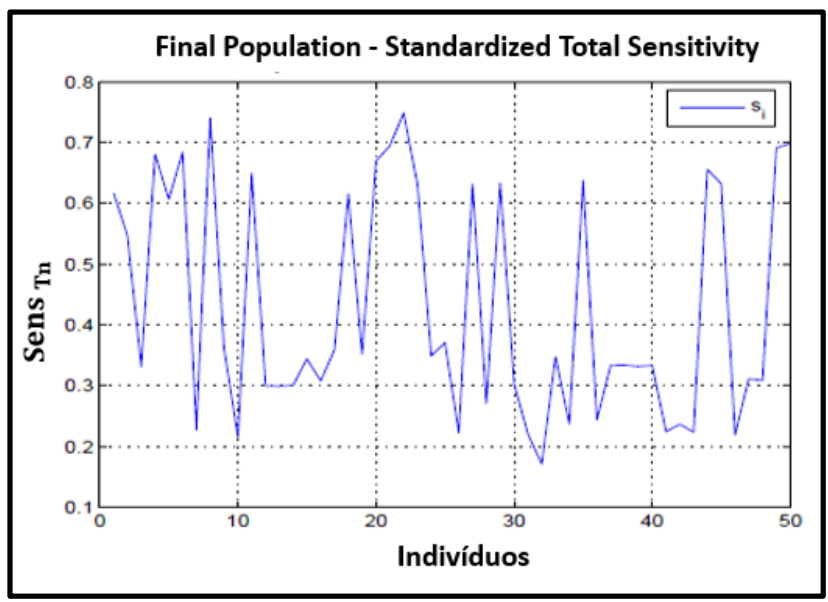

Figure 6: Total sensitivity for the final population

Below, we show the $Q$ and $R$ weighting matrices:

$\begin{aligned} Q_{L Q R} & =\left[\begin{array}{cccccc}2.59 & 0.34 & 1.13 & 0.55 & 0.35 & 1.83 \\ 0.34 & 4.39 & 1.42 & 0.29 & 1.59 & 4.57 \\ 1.13 & 1.42 & 3.49 & 5.00 & 0.39 & 0.36 \\ 0.55 & 0.29 & 5.00 & 11.15 & 0.33 & 0.36 \\ 0.35 & 1.59 & 0.39 & 0.33 & 12.34 & 0.29 \\ 1.83 & 4.57 & 0.36 & 0.36 & 0.29 & 14.93\end{array}\right] \\ R_{L Q R} & =\left[\begin{array}{ccccc}6.53 & 0.13 & 0.11 & 0.16 & 0.12 \\ 0.13 & 6.93 & 0.15 & 0.0832 & 0.13 \\ 0.11 & 0.15 & 7.45 & 0.12 & 0.15 \\ 0.16 & 0.08 & 0.12 & 9.68 & 0.13 \\ 0.12 & 0.13 & 0.15 & 0.13 & 10.56\end{array}\right]\end{aligned}$

According to (Goldberg, 1989), for the Genetic Algorithm to work effectively, it is necessary to have defined a genetic representation of the solution and a fitness function. The GA uses strategies that are expressed by fitness functions, crossover and mutation whose goal is to reach a certain maximum number of generations or a certain fitness value. To obtain these results with GA, used the fitness function that scores each individual from the satisfaction of the project specifications. According to (Coley, 1998), the use of the fitness function using the elitist method is a great alternative of implementation, because, it keeps the best individuals in the course of generations.

\subsubsection{Neural Solution - (RAE)}

Figure (7) illustrates the energy of the RAE solution and the Cholesky factor. It has as goal to present the intervals for the weights of the network where the energy is minimum. 


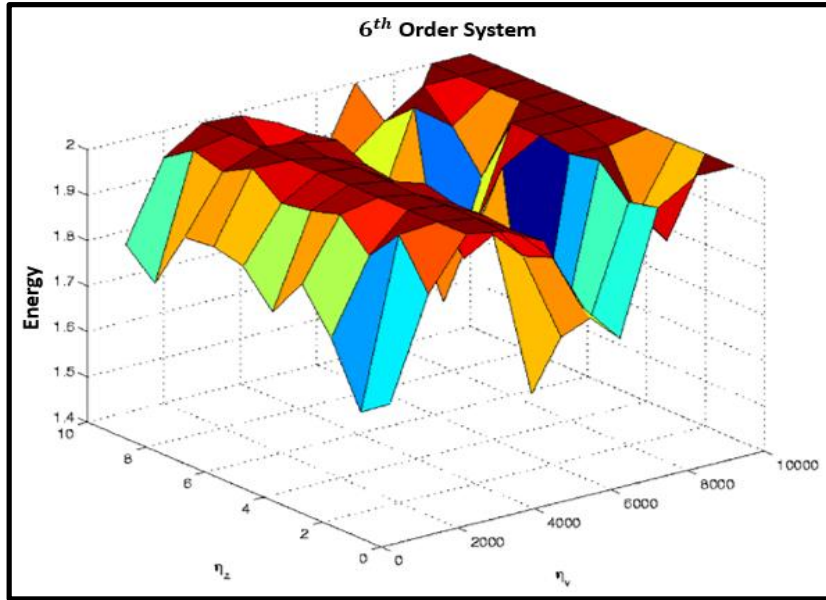

Figure 7: Energy of the RAE solution and the Cholesky factor

To measure the RAE solution, the infinity norm is used as a way to evaluate its solution

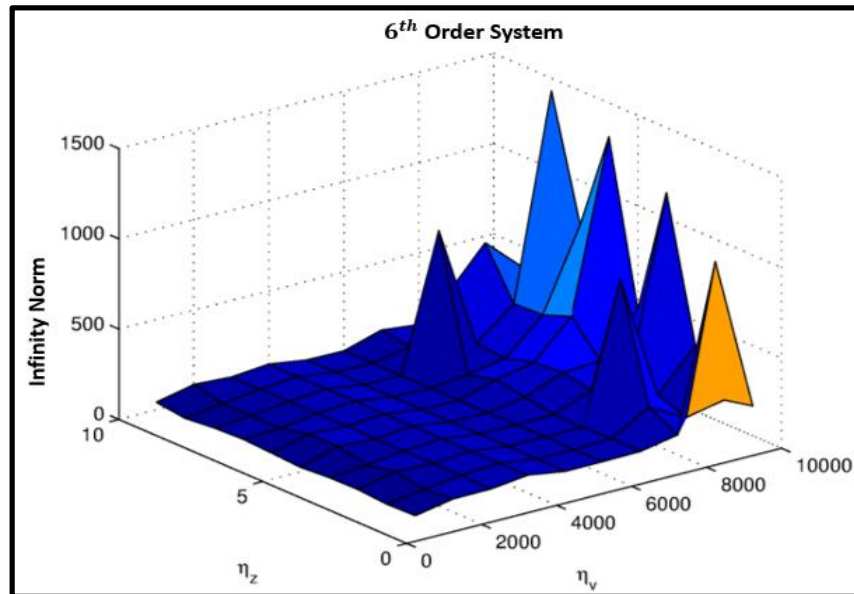

Figure 8: Infinite norm of EAR for variation parameters $\eta_{v}$ and $\eta_{z}$

Figure 8 shows the infinity norm of the network layers. The values of the standard tend towards a stable solution. The elements of the $U$ and $Y$ layers are null when $t \rightarrow \infty$. Consequently, a solution of RAE reached convergence in 200 iterations.
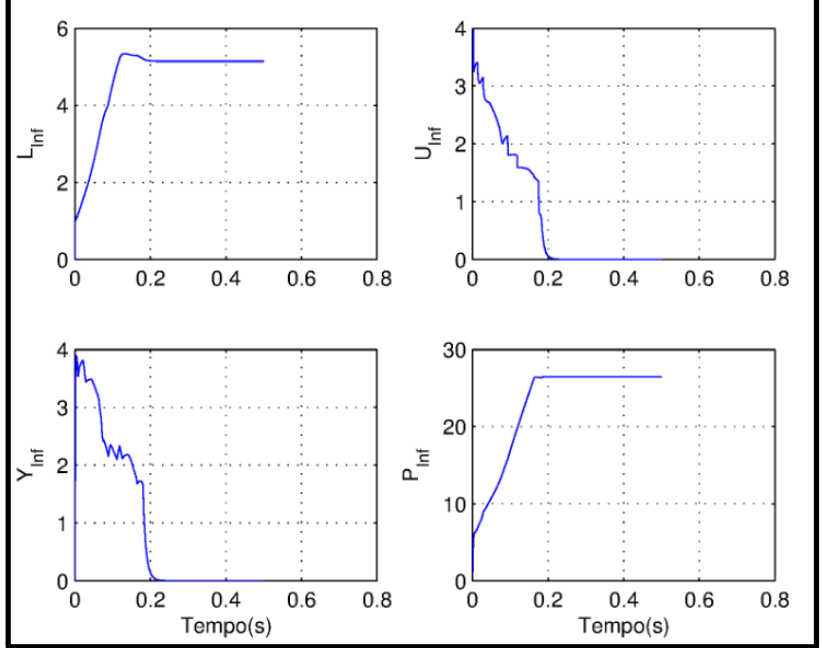

Figure 9: Infinity norm of neural network layers
As an example of a stable solution, however, which does not present RAE behavior, it is shown in figure (9) for the parameters $\eta_{v}=1000$ and $\eta_{z}=8$. As can be seen in figure (10), the infinite norms of the $U$ and $Y$ layers are accompanied by values that have an unstable norm of infinity.

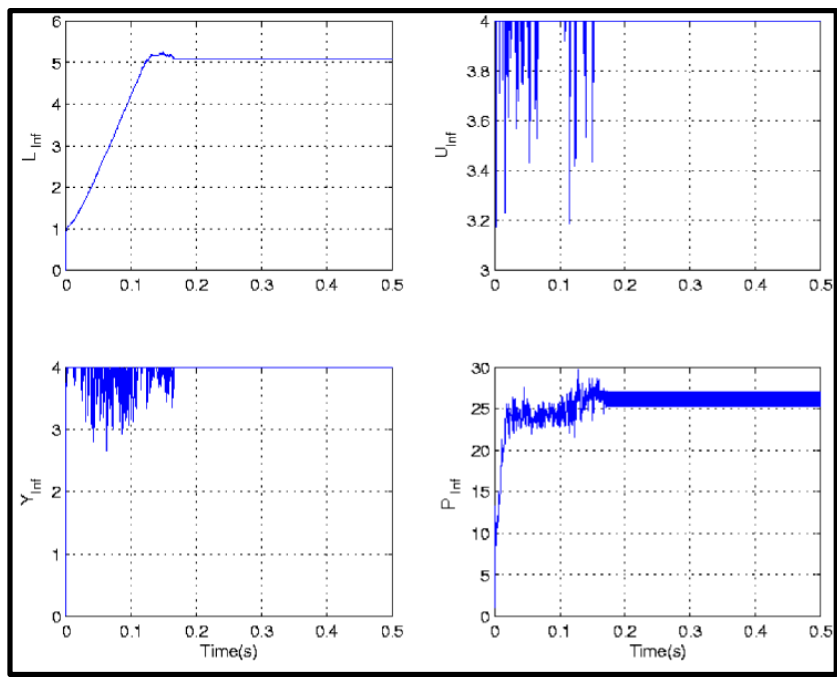

Figure 10: Infinity norm associated with solutions

The algorithm that implements the neuronal solution of the Riccati equation, consists of the implementation of the optimization structure, presented in Eq. (12), which minimizes the energy function. The $K_{R N A R}$ gain matrix obtained from the LQR using the RNAR were:

$$
K=\left[\begin{array}{rrrcrr}
-0.062 & -0.005 & -0.034 & -0.011 & -0.013 & -0.076 \\
-0.005 & -0.070 & -0.032 & 0.004 & -0.040 & -0.134 \\
-0.004 & -0.004 & -0.022 & -0.029 & -0.005 & -0.020 \\
-0.000 & -0.007 & 0.047 & 0.180 & -0.002 & -0.244 \\
0.004 & 0.012 & 0.006 & -0.001 & 0.180 & 0.012
\end{array}\right]
$$

\subsubsection{Solution via Schur method - (RAE)}

In the structure of equation (4), it has been the matrices $Q$ and $R$ that are constant and are contained in the RAE, which in turn, is solved by the Schur method. The optimal gain matrix $K_{S c h u r}$, obtained by solving the Riccati Algebraic Equation by the Schur method is given by:

$$
K=\left[\begin{array}{rrrcrr}
-0.062 & -0.005 & -0.034 & -0.011 & -0.013 & -0.076 \\
-0.005 & -0.070 & -0.032 & 0.004 & -0.040 & -0.134 \\
-0.004 & -0.004 & -0.022 & -0.029 & -0.005 & -0.020 \\
-0.000 & -0.007 & 0.047 & 0.180 & -0.002 & -0.244 \\
0.004 & 0.012 & 0.006 & -0.001 & 0.180 & 0.012
\end{array}\right]
$$

The eigenvalues obtained from the dynamic system with LQR controller are:

$$
\text { [-56.62, }-40.20,-22.00,-18.67,-13.98 \pm j 1.86]
$$

Again, the SVD is used for analysis in the frequency domain of the system with LQR, where the elements of the matrix $S$ of singular values obtained are:

$$
\text { [56.66, 40.22, 33.52, 21.99, 19.12, 5.79] }
$$

Analyzing the two results by the Schur method and by the RNA, see that both are well approximated, and the artificial neural network are more stable, since this difference becomes imperceptible, because it is in the decimal precision of the solutions after eighth decimal place. It is observed that the eigenvalues of the system and the diagonals of the $Q$ and $R$ matrices directly influence the performance and convergence of the RNA, so that the values of the $Q$ and $R$ matrices can be 
reduced or increased uniformly, thus improving the RAE solution.

Being made an analysis in the adjustments of the constants $\eta_{v}$ and $\eta_{z}$, see that the found results act with that speed the algorithm will converge or it will diverge and the as a solution can be optimal. The analysis of the results of the RAE solution via RNAR can be observed in figures (7) and (8) which are based on the behavior of the output layer, considering the input and the hidden layers. The convergence analysis leads to two aspects of the RAE solution, the first regarding the existence of a single solution, and the second focuses on the speed of the neural network in reaching the value of the solution at steady state. Figure 7 shows the results of the infinity norm of the layers $L, U, Y(t)$ and $P(t)$, and figure (8) shows the results of the norm of infinity associated with the respective solutions.

This analysis has as main objective to verify two types of results the first one is related to the best fit of the constants $\eta_{v}$ and $\eta_{z}$ and the second begins the choice of the best initial condition, and a another point to consider is the number of iterations used to compute the solution Riccati Algebraic Equation. Figure (9) shows the surface of the energy function with adjustments of the parameters $\eta_{v}$ and $\eta_{z}$.

\section{CONCLUSION}

After analyzing the parameters and obtaining the $Q$ and $R$ matrices presented by the proposed methodology of geneticneural fusion, it can be observed that the hybrid model presented satisfactory results on the surfaces, metrics of the norm of infinity and decomposition in singular values, according to the analysis of the performance of the wind power generation system, with respect to the optimal control problem. As future work, about the proposed recurrent neural network, one can analyze the algorithmic complexity of this RNAR, as well as, to study the complexity of the neural network defining the problem size, aiming to study the computing time needed for the resolution of the Riccati Algebraic Equation based on the worst-case study. Another alternative as a proposal to be implemented and compared with the neural-genetic model in future works is the use of SVM, the Support Vector Machine, in order to find the best hyperplane between the analyzed data, seeking to maximize the distance between the points next, and with that, find the optimal gain of the plant. However, from the results achieved in this article, we conclude that new intelligent computational approaches can be implemented and promising results are expected in the most varied real-time project application and system control.

\section{REFERENCES}

[1] Athans, Michael e Falb, L. P. (1966). Optimal Control-An Introduction to the Theory and Its Apllications, McGRAW-Hill Book Company, United States of America.

[2] Coley, David A. (1999). An Introduction to Genetic Algorithms for scientists and Engineers: World Scientific Publishing, 1999.

[3] Goldberg, D. Genetic Algorithms in Search, Optimization, and Machine Learning. Reading: Addison Wesley, 1989.

[4] Guang Wu e Wang. J. A multilayer recurrent neural network for solving continuous-time algebraic Riccati equation, Neural network (1998).

[5] Hansen, A. D., Jauch, C. Sorensen, P. Iov e F.,Blaabjerg (2003). Dynamic wind turbine models in power system simulation tool, Riso national Laboratory.

[6] Laub, A. J. (1979). A schur method for solving algebraic riccati equations, IEEE Transactions on Automatic Control 24(6): 913:921.

[7] Myoen-Song Choi, J.-W. L. e. K. L. (1996). An auxiliary lqg/ltr robust controller design for cogeneration plants young-moon park, Energy Conversion, IEEE Transactions on 1(2): 407-413.

[8] Murata, T.; Muyeen, S.M; Tamura, J. Stability Augmentation of a Grid-connected Wind Farm. Hokkaido: Springer, 2009.

[9] Pinto, V. P. (2007). Modelagem e Simulação de uma Planta Eólica Controlada por um Regulador Linear Quadrático Conectada ao Sistema de Distribuição de Energia Elétrica, $\mathrm{PhD}$ tese, Universidade Federal do Ceará.

[10] Silva Abreu, Ivanildo (2008). Controle inteligente LQR neuro - genético para alocação de auto-estrutura, Tese de Doutorado, Pós-Graduação em Engenharia Elétrica, UFPA.

[11] Viva, Safont. J. (2016). Simulador de Generador Eólico Doblemente Alimentado. Tese de Doutorado. Universitat Politècnica de València 